\title{
Providers, Prescriptions and Patients: Methodology for U.S. Health Care Reform
}

\author{
Peter Cabeceiras ${ }^{\mathrm{a}}$
}

\begin{abstract}
Health care reform should be considered equal parts moral and economic issues. The United States spends more on health care than any other nation in the world (\$2.6 trillion annually), yet the U.S. has some of the worst health outcomes of developed nations. ${ }^{1,2}$ Although health care spending comprises over one-sixth of the economy (17.9\% of GDP), over fifty million U.S. citizens live without health insurance. The U.S. needs to use finances more efficaciously not only to enhance quality of care and patient outcomes, but to avoid a looming economic crisis. ${ }^{3}$ Recently, the Congressional Budget Office reported that if Medicare cost inflation exceeds overall economic growth by $2.5 \%$, the top marginal tax rates in 2050 will approach $92 \%$. $^{3,4}$ The Patient Protection and Affordable Care Act (ACA) addresses problems associated with cost, coverage and quality by introducing: the Individual Mandate, Accountable Care Organizations (ACOs), Bundled Payments for Care Improvement (BPCI) Initiative, Patient-Centered Outcomes Research Institute (PCORI), and many more pilot programs that act as vehicles of site-specific reform. However, underlying health care problems still remain that must be resolved to facilitate the ACA reforms. These daunting issues include, but are certainly not limited to: the shortage of physicians, ACO practitioner liability, weaknesses of the Individual Mandate, the remaining uninsured and the outmoded practices of the U.S. Food and Drug Administration (FDA). The goal of this paper is to address these problems and propose strategic reforms that will either augment or exist concomitantly with the ACA.
\end{abstract}

Keywords: Healthcare; Affordable Care Act

\section{The Dearth of Physicians Should Be Addressed and Remedied}

The Association of American Medical Colleges projects increasing shortages in nearly all physician specialties and subgroups. It is estimated that by 2020 , shortages of doctors will reach 46,000+ in surgical and other specialties, 4,000 in oncology alone and 45,000-65,000 in primary care. ${ }^{5}$ The lack of practitioners will be concentrated in specialties that treat older adults; this problem is further augmented as 10,000 baby boomers turn 65 each day, and one-third of practicing physicians plan on retiring over the course of the next decade. $^{5}$ The current supply of physicians is so far behind the demand that it is common for specialties to only fill one-third of their residency programs.

Increasing the population of doctors is a solution at the crux of health care reform. In 2014, over 30 million Americans will gain access to health insurance, and subsequently health care, yet there is already a severe undersupply of physicians. When there are not enough practitioners, having health coverage does not necessarily imply that one has access to health care. The federal government should be funding the creation of new medical schools. In order to get more medical students involved in primary care, instead of other more lucrative specialties, medical schools that only teach primary care physicians (PCPs) need to be established as well. ${ }^{6}$ It is common for medical students to graduate with over $\$ 200,000$ in debt, so there should be a slew of programs that prospective medical students can enter to receive medical school tuition assistance. ${ }^{7}$ The number of nurse practitioners and physician assistants must also increase because they can be trained more expeditiously than physicians can, and they have the potential to accommodate patient needs and lower waiting times. The main tenets of the ACA are to elevate access to and affordability of care, but these principles will be sacrificed if care providers are too few in number to administer care to the enormous forthcoming influx of patients.

\section{The Emergency Department}

The volume of patients visiting the emergency department (ED) must be significantly reduced. The ACA reforms intend to assuage the overuse of the ED by directing people to instead use primary care services when appropriate. ${ }^{8}$ However, the National Association of Community Health Centers estimates that there are already over 56 million Americans without sufficient access to PCPs. EDs with a high volume of patients become overcrowded, and patients who need urgent attention are resigned to wait. Many patients at the ED either have problems better suited for PCPs, or have waited long enough for these problems to develop into emergencies. Even more concerning, the issue of overcrowding in EDs gets inadvertently compounded when the concept of frequent visitors is taken into account. When the bulk of an ED patient's condition is ameliorated, that patient is discharged from the ED and usually encouraged to see another specialist to prevent a relapse of the same condition. However, most people do not or cannot seek a specialist, nor gain control over their ailments, so they end up revisiting the ED every time their symptoms reach unbearable levels. This creates a persistent cycle in which the patient cannot sustain good health, resulting in more and more visits to the ED and a growing dependence on having his or her disease managed rather than prevented.

To elevate quality of care, the volume of patients seeking ED treatment must be scaled down to include those patients who truly need emergency care. The ACA will not significantly affect the trend of inpatient and outpatient ED use, ${ }^{9}$ so coordination of care is necessary to efficaciously direct patients who do not need urgent care to the specialist apposite for treating their particular medical condition. The specialties of the medical fields are vast and variegated, which 
is why people make the simple-yet often incorrect and detrimental-decision to go the ED much too frequently. A system must be created that lists symptoms or conditions that require medical attention, and then connects these to the optimal specialty of physician for the job. It would be similar to the diagnosis-related group (DRG) system in that the DRG system connects procedures to the appropriate prices, whereas this system would connect a patient's symptoms to the appropriate physician(s). Once this optimized treatment list (OTL) is completed by a board of thoughtful and deliberative physicians, it can be incorporated into health insurance plans to educate policy holders about which specialties on the list are covered in their specific plan. Eventually the OTL can become a tool that health insurers use to advertise how their plan is superior to other plans in the exchanges, and this will enable widespread adoption and dissemination of the OTL. The more people know about the OTL, the more people can make better decisions as to whom they should visit for their medical conditions. The OTL can help to catalyze the rapid flux in American health care culture from primarily managing disease, to balancing disease management and prevention.

\section{Prevention Services}

The current U.S. health care system is a fee-for-service model that focuses on disease management. The notion of health care should be an embodiment of disease prevention and management. The Patient Centered Medical Home (PCMH) refers to a physician's office that emphasizes preventative care, instead of reacting to a patient who is already sick. In the effort of providing economical care, nurse practitioners and physician assistants provide services that do not require the skills of a physician. PCMHs incentivize economical care because they employ a form of capitation. Instead of being paid for every patient encounter, a PCMH is given a lump sum based on the amount of patients and a time frame. The PCMH keeps the money that it does not spend after a certain period of time, and this incentivizes the PCMH to keep patients healthy through preventative services. PCMHs should be integrated into hospitals across the country because they introduce preventative care at the clinical level. ${ }^{7}$

Disease prevention should also be incentivized in everyday life by more implementation of employee health and wellness incentives. According to Towers Watson, employee health incentives are not a new idea, yet only $25 \%$ of employers awarded incentives based on biometric outcomes in $2011 .{ }^{10} \mathrm{~A}$ maximum of $30 \%$ (50\% for smokers) of an employee's premium can be set as the amount employees receive as an award, or lose as a penalty, for annual biometric outcomes in $2014{ }^{10}$ Some employees who are relatively unhealthy may find it more difficult to attain these extrinsic incentives, so there must be measures taken to prevent feelings of alienation. Rewards and incentives should take into account relative levels of employee health in order to create goals that are personalized and attainable for everyone. ${ }^{1}$

In order for wellness incentives to motivate employees, outcome-based incentives must also be paired with progressbased incentives. Small progress-based incentives award incremental improvements while encouraging progress towards the largest outcome-based award. However, the amount of the reward is not always a predictor of healthy

\footnotetext{
${ }^{1}$ A body mass index (BMI) can be used to set goals that are fair and personalized for each employee.
}

changes. Having larger incentives allows for more creative employee programs, but the goal is to get people to stay healthy even after they receive their reward. A behavioral economist might even suggest an approach in which employees do not know what incentives are in store for them if they participate in a program. ${ }^{10}$ If they make progress in a program, and receive benefits without expecting that they were going to receive benefits, they might be more likely to continue changing their behavior constructively. ${ }^{10}$

To summarize, more employers should be helping to prevent disease by facilitating for a healthier workplace culture.

Incentives programs should include:

a. A large outcome-based reward that is tailored for each employee

b. Small progress-based rewards

c. Progress and outcome-based rewards that is initially unbeknownst to employees

\section{Accountable Care Organizations Should Protect Physicians from Liability}

Convoluted health care payment methods, including billing, bundling, negotiated costs and reductions, and forced payment reductions such as with Medicare and Medicaid payments ${ }^{2}$ raise the difficultly of identifying and abating the costs driving health care inflation. It is important that all practitioners have an infrastructure for making cost-effective decisions because the practitioner is the primary determinant of health care cost; the practitioner ultimately makes the decisions regarding type and quantity of care the patient will be receiving. ${ }^{11}$

Physicians now have an enhanced power to restructure aspects of treatment and post-hospital care under new Accountable Care Organizations (ACOs). ${ }^{12}$ An ACO is a network of health care providers (usually from the same hospital) that works towards reducing the costs of medical services. These organizations have the potential to increase physician autonomy by encouraging them to create economical treatment plans, but the functionality of the ACOs is crippled by the significant liabilities that physicians face. Liability can leave practitioners unreasonably open to malpractice lawsuits. If a patient has an unfavorable outcome, whether it was the fault of the physician or not, the physician can be found culpable. ${ }^{13}$

The problem with medical liability has been an enduring issue that complicates the life of a physician and slows the delivery of care. There already exist expert guidelines for medical treatment, but they merely act as a source of information. The solution to the issue of liability hindering the progress of ACOs is to grant legal protection to physicians who practice under expert guidelines. Of course,

\footnotetext{
${ }^{2}$ Historically, payments from insurance companies as well as the Centers for Medicare and Medicaid Services have been erroneously labeled "reimbursement." By definition, though, almost all of these monies represent payments for services rendered and include consideration of provider time, case difficulty, and physical facility costs. A "reimbursement" is payment for monies spent, which would be the appropriate term if the patient initially paid for the medical service and was then "made whole" by being recompensed for this expense. But when paid by the insurer directly to the deliverer of the service, the term "reimbursement" is incorrect.
} 
this assumes that the patient has no contrary indications to following the guidelines. Since there can be a slew of unforeseen events that may or may not be addressed in the guidelines, there should be appropriate liability caps imposed for procedures. The practice of medicine is a continual experiment, and expert guidelines provide information on which practices have the highest likelihood of success based on previous experiences and research. If physicians have protection under these guidelines, they can abrogate the excessive tests and procedures that are done primarily to lower liability.

\section{The Adverse Overutilization of Modern Medicine}

One typical overuse of medicine that can be partly attributed to liability protection is when a middle-aged patient has chest pain and goes to their doctor. Fifteen years ago, if the patient had a normal EKG and the pain receded, the average doctor would discharge the patient. Nowadays, the patient could receive a stress test, an echocardiogram, a mobile Holter monitor, and possibly a cardiac catheterization; even though these services might be unnecessary and detrimental for the patient. ${ }^{14}$ In the case of the stress test, the criteria for performing the test are arbitrary, and the test results are not definitive and tend to necessitate the additional aforementioned services. ${ }^{15}$ All of these extra precautions can provide liability protection to physicians, and doctors generate income when they provide these services, so incentives (ACOs sharing savings with physicians), in addition to preestablished liability protection, are required if physicians are to usher in a new era of cost-effective, value-based care.

Liability protection, in addition to physician teamwork, can improve how common procedures, like cardiac catheterizations are implemented. A cardiac catheterization is an invasive procedure for detecting obstructive coronary artery disease (CAD). The criteria used to justify performing this procedure include demonstrating that the patient has a high risk for obstructive CAD through a classification of chest pain, and high-risk results from a stress test. ${ }^{16}$ A recent investigation compared the amount of cardiac catheterizations performed in New York State to those performed in Ontario, Canada. Compared to Ontario, New York State had twice as many low risk patients undergoing the procedure, a lower percentage of obstructive CAD observed and a slightly higher 30 day mortality rate. ${ }^{16}$ If New York State physicians could adopt more selective criteria as to which patients truly need cardiac catheterization, they could potentially save $\$ 75$ million annually and lower patient mortality. ${ }^{16}$ Although it may be intuitive to be against an ACO providing less medical care, there are many examples like the aforementioned where less medical treatment leads to better outcomes. ${ }^{17}$

\section{The Individual Mandate Can Be Strengthened and Stabilized}

In terms of fairness, people who can afford health insurance have a societal obligation to buy health insurance (as long as they do not have religious or other special objections). Premiums can be kept at a manageable level when a balance is maintained between healthy and sick people from all age groups. However, some people exploit the health insurance system by only insuring themselves when they need medical services. Hence the funds that society has been depositing into insurance (premiums) help to pay for the health care of individuals who have not contributed any monies of their own. The net effect of this opportunistic insuring is increased insurance costs due to more people filing claims. The ACA created the individual mandate to increase the number of people paying into insurance, and to combat the issue of the transient insured in order to lower and stabilize the cost of insurance. ${ }^{18,19}$

\section{The Penalty Fee}

The ACA mandates that people who do not have health insurance will pay a penalty fee; unfortunately, that penalty may be negligible. ${ }^{20,21}$ For some people in good health, it will still be cheaper to remain uninsured and pay a penalty fee than to pay into health insurance. It is projected that 2-3 million adults who can afford private insurance plans will opt to pay the penalty instead of insuring themselves. ${ }^{21}$ This raises the percentage of people in health insurance plans who file claims for their health problems, causing an inevitable rise in the costs of premiums. Private insurers anticipating this problem have been proactively raising premiums before health insurance exchanges become active. ${ }^{22}$ In order to deter individuals from opportunistically insuring, there should be a penalty fee for uninsured adults who can afford to insure themselves that is more expensive than the ACA bronze level plan according to the individual's demographic (IRS information, employment status and available insurance offerings/eligibility). Although this reform will generate a fair amount of pushback, it will incentivize the purchase of the basal level of health insurance policy, and costs will be more widely distributed, thus lowering costs for everyone.

\section{The Remaining Uninsured Need Better Options}

According to the congressional Budget Office, the ACA will bring the percentage of American residents with health insurance up to $90 \%{ }^{23}$ That leaves around 19 million American adults (non-elderly) without health insurance after reform. ${ }^{21}$ The Urban Institute estimates this population subset to be comprised of five groups:

1. 7.03 million young adults eligible for, but not enrolled in Medicaid

2. $\quad 1.52$ million young adults eligible for subsidies in the health insurance exchanges

3. 3.04 million older adults with no economical option for health insurance due to low income, and therefore exempt from the individual mandate

4. 4.75 million undocumented immigrants

5. 2.85 million wealthy adults who have an affordable private option, but choose to pay the mandate penalty rather than become insured

Since health insurance coverage is associated with better health, ${ }^{24}$ and a large and diverse group of people participating in insurance helps to preserve manageable costs, there should be measures taken to insure as many Americans as possible. States should not be allowed to opt out of the Medicaid expansion brought forth by the ACA. States, like Texas, that have opted out of the Medicaid expansion will still have a tremendous amount of people without health insurance post$2014 .^{7}$ 


\section{Undocumented Immigrants}

The question remains: should undocumented immigrants have expanded access to health care, and if so, how much? Before answering this question, it would be instructive to first explore the significant, yet often obscured, impact that this particular cohort has on the U.S. health care system. Undocumented immigrants and U.S. citizens account for $\$ 15.4$ billion and $\$ 1$ trillion respectively in annual health care expenditures. $^{25}$ In 2014, unauthorized immigrants will be verboten to purchase private or public forms of health insurance in the state exchanges; however they are exempt from the individual mandate, so they will not be penalized for being uninsured. Undocumented immigrants must either pay out of pocket, purchase private insurance outside of exchanges or get uncompensated care in emergency departments. ED care of indigent patients is required by the Emergency Medical Treatment and Active Labor Act of 1986; $50 \%$ of unauthorized immigrants receive uncompensated care, and Medicaid pays for the other $50 \%$. $^{25}$ The ED care of nonnaturalized indigent patients puts an annual financial strain of $\$ 250$ million on the federal government because hospitals must be remunerated for the care they provided for free. ${ }^{26}$ However, the cost of paying for immigrant ED care is less than that of subsidizing their purchase of health insurance, so it is unfeasible to reform low-income immigrant access to health care through government subsidies for insurance. The same sentiment is highlighted by the popular view that taxpayers are the only individuals who should be eligible for government subsidies in health insurance exchanges; the government cannot afford to grant subsidies to all unauthorized migrants in addition to U.S. citizens. Since federal subsidies are out of the question, there remain two options for improving access to health care for undocumented immigrants without additional federal spending.

The first area of reform pertains to the low-income unauthorized immigrants who rely on the ED for their medical needs. In the interest of the public health of the United States, in addition to ED services, measures for the prevention and treatment of infectious diseases should be covered for undocumented immigrants. ${ }^{25}$ Considering experiences from past pandemics, this can actually exist as a preventative medicine, cost-saving entity. The H1N1 virus outbreak in 2009 prompted the federal government to spend $\$ 1$ billion in producing vaccines alone, a single expenditure that dwarfs the annual cost of immigrant ED care. ${ }^{27}$ A modest increase in state funding of preventative medicine for undocumented migrants could help to avoid the drastically expensive measures associated with large outbreaks of disease.

The Border Security, Economic Opportunity, and Immigration Modernization Act that has had recent success in the U.S. Senate yields potential for further health reforms. This legislation champions the idea of a ten year pathway to citizenship, with the assumption that an undocumented immigrant on this path to citizenship is employed during their stay in the U.S. Although they are working, undocumented immigrants are forbidden to be officially employed by the Immigration Reform and Control Act (IRCA) of 1986. As evidenced by millions of undocumented workers being unofficially employed in the U.S., IRCA has not achieved its objectives of deterring illegal immigration, and has actually served to push a large population into the shadows where they can be exploited without protection. ${ }^{28}$ Furthermore, the verification constructs used to demonstrate citizenship to employers (I-9 form, driver's license and social security card) can be counterfeited inexpensively. ${ }^{29}$ Therein lies the grounds for revoking IRCA, making it legal, just as it was pre-1986, to employ undocumented immigrants. In doing so, undocumented immigrants on the ten year pathway to citizenship will be eligible for non-subsidized, employercovered health insurance. By recognizing undocumented immigrant workers for their contributions, and granting them eligibility to employer-based coverage, the United States is incentivizing adherence to the ten year regularization pathway while helping to keep immigrants healthy along the way.

In regards to undocumented immigrants who can afford to buy their own health insurance, their options post-ACA are limited to purchasing private coverage outside of insurance exchanges; a market that will be invariably limited. These undocumented immigrants should be allowed to purchase private insurance inside and outside of the health insurance exchanges because they are not receiving any government subsidies and can possibly help to lower premiums for everyone. Premium costs decline when there are more healthy people in a health insurance plan, and undocumented immigrants of Hispanic origin have actually been shown to be healthier and have lower adult mortality rates than U.S. citizens. $^{25}$

\section{The FDA Should Be Optimized for the $21^{\text {st }}$ Century}

\section{General Patient Safety}

The ACA has many provisions for maintaining patient safety, but they are too vague and far-reaching to hone in on specific problems, like the issues with "black box" drug warnings. The FDA issues "black box" drug warnings for potentially dangerous medications that can still be used in practice. More dependable, available options should be used instead of drugs with "black box" warnings. The decision to administer the safer drug seems like palpable logic, but the converse transpires much too often without medical justification. One example in particular involves a plasma expander named Pentastarch (Pentaspan). Recently, the FDA issued a "black box" warning on this drug because of studies connecting the drug to renal failure in patients. ${ }^{30}$ Even though simple crystalloid solution can be used instead of Pentaspan, and Pentaspan is more expensive than saline, Pentaspan continues to be used in some of the top hospitals in the country. Understandably, medical care providers are too busy to keep up with the literature for every drug in existence. Since this problem resides at the individual hospital level, there should be a financial incentives package that goes to the venerable physicians who expose misuse of "black box drugs" to departments that can restrict access to these drugs, like the Pharmacy department. The package, or prize, can be in the form of a federal tax break, because by assuring that safer drugs are being used, Medicaid and Medicare save money that would have been spent on future patient complications.

\section{Molecular Medicine and Oncology}

Although there have been many recent advances in therapeutics, there are still a multitude of diseases that have no effective remedy. New genomic technologies have allowed rapid production of therapeutic breakthroughs based on specific gene and protein functional interactions in arcane 
diseases, like Autism and cancer. Unlike conventional "one size fits all" medicine, these drugs treat the individual's disease; these drugs are the nascent stages of personalized, molecular medicine. Millions of patients who have untreatable diseases need access to the discoveries of modern medicine, but these drugs must first be tested for safety and efficacy by the FDA before they can be allowed on the market. Unfortunately, the FDA often stifles innovations with its archaic IT systems, limited influence, wait times and emphasis on statistics over individual outcomes. ${ }^{31}$ Thus, many patients who need new medicines are resigned to endure the FDA's shortcomings. Without sacrificing safety and efficacy standards, reforms for the FDA should emphasize faster approval for breakthrough therapeutics and updated protocols to better facilitate for the future of medicine; primarily in oncology.

$50 \%$ of men and women are projected to be diagnosed with some form of cancer over their lifetime. If oncologists and their patients want to utilize personalized medicine, then a myriad of drugs that target specific biomarkers must be developed. Unfortunately, the U.S. cancer drug discovery and development process is a dreadful failure. It takes 7-12 years from research to drug approval, over $\$ 1$ billion is spent on each candidate drug, and only about $5 \%$ of these drugs successfully reach the market. ${ }^{3}$

Why do these cancer drugs fail? In FDA randomized clinical trials, drugs (and placebos) are given to an idiosyncratic group of patients; all patients have different needs (there are perhaps 200 different types of diseases we call cancer). Thus, more often than not, no significant results emerge, and the drug fails even though particular individuals have beneficial outcomes. The patients who experienced better outcomes have cancers with specific biomarkers, like cytokines, that the drug happens to target; the drug is engineered for a very specific subpopulation of patients, yet it fails the FDA evaluation. This is the paradoxical example of how the FDA investigatory protocols are precluding the advancement of personalized medicine in oncology. To circumvent this hindrance, pharmaceutical companies must clearly define the individuals that their drug is intended to treat, and the FDA needs to choose patients for clinical trials based on genomic profiles. At its core, cancer is a genomic disease that requires therapeutics at the genomic level. The rationale behind approving a drug only for a very specific group of patients is that development costs will decline, and the approval process will be shorter because fewer patient subtypes need to be tested by the FDA.

\section{Late-Onset Diseases}

In regards to late-onset diseases, some drugs have the potential to meet unmet medical needs, but cannot be approved due to the unreasonable stringency of the FDA. The FDA often cannot demonstrate efficacy of drugs for late-onset diseases because it takes 10-30 years for patients to develop an outcome upon which to infer; an infeasible, if not

\footnotetext{
${ }^{3}$ The gap between breakthrough research discovery, the publication and the approval of a drug is cleverly dubbed "the valley of death." Out of all of the stages in the translational pipeline, there is a $50 \%$ failure rate at the late stage, phase III clinical trials(the most expensive process). Intriguingly, these drugs pass phase I and II clinical trials; it is not until phase III that they fail due to a lack of efficacy.
}

impossible, span of time for a set of clinical trials. The FDA can make progress in drug approval for long-term diseases by allowing reductions in endpoints that are based on improvements, rather than a distant outcome. This proposed reform should be an amendment to the 1992 Accelerated Approval Rule that allows for a "surrogate marker" of improvement to replace unattainable outcomes as the grounds for approval of drugs for late-onset diseases, especially when there is great unmet medical need. ${ }^{4}$ Even though accelerating drug approval raises the chances that risks are overlooked, latent risks are not uncovered in the short studies of the FDA. Once "surrogate markers" of significant improvement have been identified, the benefits of getting the much needed therapeutic out on the market outweigh the uncertainty; and post-market surveillance can ensure that latent risks are promptly discovered if they do arise. The FDA must be strategic with how it implements its protocols, because their inflexibility can deter the creation of drugs that satisfy unmet medical needs.

\section{510(K) Clearance Process}

The Food, Drug, and Cosmetics Act of 1976 introduced two methods for the FDA to regulate medical devices. The FDA tests medical devices for safety and efficacy by the process of premarket approval (PMA). The 510(K) clearance is an alternate route that allows medical devices to reach the market by circumventing FDA approval. If a device has "substantial equivalence" to a device already on the market (a predicate), then it can also be cleared, not approved, by the FDA $510(\mathrm{k})$ process. The $510(\mathrm{~K})$ clearance allows the device to gain access to the market. ${ }^{32}$ In 1976 , most medical devices were simplistic, non-implantable and non-life sustaining; the $510(\mathrm{~K})$ clearance was created to clear these types of devices. As devices became more complex, and central to the lives of patients, the FDA lost the ability to assess safety and efficacy of devices because of deregulations contained within the Medical Device User Fee and Modernization Act of 2002. Post-2002, in only $1 \%$ of cases has the FDA actually investigated the safety and efficacy of medical devices through PMA. ${ }^{32}$ As a result, $78 \%$ of recalled medical devices from 2005 to 2009 were devices which had been cleared via the $510(\mathrm{~K})$ process. $^{32}$ The $510(\mathrm{~K})$ process is an antiquated review process that was designed for uncomplicated devices, yet it is being used on some of the most high-risk devices seeking access to the market. The more stringent PMA evaluation should be the only evaluation used on medical devices with moderate to high risk because a predicate is not necessarily an assurance of safety of a completely different device.

\section{Dietary Supplements}

The FDA has scant jurisdiction over dietary supplements. The 1994 Dietary Supplement Health and Education Act does not require supplements to be proven effective, or safe. This

\footnotetext{
${ }^{4}$ Around 12 million people are afflicted with polycystic kidney disease (PKD), a genetic disorder that results in total renal failure. The only way that the current FDA can evaluate the effectiveness of a drug for PKD is to compare how many patients taking a drug or placebo eventually have renal failure; but it takes around 30 years to incur renal failure. The "surrogate marker" of amelioration would be a reduction in cysts, because the cysts cause renal failure.
} 
has led to consumers purchasing athletic and nutritional supplements containing compounds that have been previously taken off the pharmaceutical market due to safety concerns, like DMAA (1,3-dimethylamyl-amine). ${ }^{33}$ DMAA has been shown to significantly increase blood pressure and risk of heart attack. In 2010, U.S. consumers spent over $\$ 100$ million on DMAA-containing supplements. ${ }^{33}$ With athletic supplements and holistic medicines becoming increasingly popular, the FDA needs the authority to protect consumers by evaluating safety and efficacy of supplements, before providing its imprimatur.

\section{References}

1. Onie R, Farmer, Paul., \& Behforouz, Heidi. Realigning Health with Care. Stanford Social Innovation Review. 2012.

2. Murray CJ, Frenk J. Ranking 37th-measuring the performance of the US health care system. New England Journal of Medicine. 2010;362(2):98-99.

3. Chernew ME, Baicker K, Hsu J. The specter of financial armageddon - health care and federal debt in the United States. New England Journal of Medicine. 2010;362(13):1166-1168.

4. Chandra A. Controling the Cost of Health Care in the United States [Internet]. Harvard Kennedy School; 2013. Podcast

5. Darves B. Physician Shortages in the Specialties Taking a Toll. New England Journal of Medicine. 2011.

6. Cohen J. New Med School Aims To Train Primary Care Docs. Kaiser Health News. 2013.

7. Zwelling L. Expert Insights. In: Cabeceiras P, ed. Division of Cancer Medicine: UT MD Anderson Cancer Center; 2013.

8. Siegel B. Primary Care, Improved ED Throughput Are Keys to Reducing ED Overcrowding and Preparing for ACA Implementation. NAPH. February 2013 2013:1-8.

9. Chen C, Scheffler G, Chandra A. Massachusetts' health care reform and emergency department utilization. New England Journal of Medicine. 2011;365(12).

10. Donovan P. Health and Wellness Incentives: Positioning for Outcome-Based Rewards [Internet]; 2013. Podcast: 12:22

11. Fuchs VR. Economics, values, and health care reform. American Economic Review. 1996;86(1):1-24.

12. Emanuel EJ, Pearson SD. Physician autonomy and health care reform. JAMA: The Journal of the American Medical Association. 2012;307(4):367-368.

13. Harvey HB, Cohen IG. The Looming Threat of Liability for Accountable Care Organizations and What to Do About It. JAMA : the journal of the American Medical Association. 2013:1-2.

14. Gawande A. The Cost Conundrum: What a Texas town can teach us about health care. The New Yorker 2009.

15. Rozner M. Expert Insights. In: Cabeceiras P, ed. Dept. of Anesthesiology \& Perioperative Medicine: UT MD Anderson Cancer Center; 2013.
16. Ko DT, Tu JV, Austin PC, et al. Prevalence and Extent of Obstructive Coronary Artery Disease Among Patients Undergoing Elective Coronary Catheterization in New York State and OntarioObstructive CAD and Coronary CatheterizationObstructive $\mathrm{CAD}$ and Coronary Catheterization. JAMA : the journal of the American Medical Association. 2013;310(2):163-169.

17. Weinstein MC, Skinner JA. Comparative effectiveness and health care spending - implications for reform. New England Journal of Medicine. 2010;362(5):460-465.

18. The Patient Protection and Affordable Care Act. Public Law. 2010.

19. Arrow K, Auerbach A, Bertko J, et al. Toward a 21stcentury health care system: recommendations for health care reform. Annals of Internal Medicine. 2009;150(7):493-495.

20. Starr P. Remedy and Reaction: The Peculiar American Struggle over Health Care Reform [Internet]; 2011. Podcast: 16:52

21. Buettgens M, Hall M. Who will be uninsured after health insurance reform. Robert Wood Johnson Foundation. 2011.

22. Emanuel E. Health-Care Exchanges Will Need the Young Invincibles The Wall Street Journal. 2013.

23. Taxation. CBOsotJCo. CBO's February 2013 Estimate of the Effects of the Affordable Care Act on Health Insurance Coverage. 2013.

24. Kilbourne AM. Care without coverage: too little, too late. Journal of the National Medical Association. 2005;97(11):1578.

25. Stimpson JP, Wilson FA, Su D. Unauthorized Immigrants Spend Less Than Other Immigrants And US Natives On Health Care. Health Affairs. 2013.

26. Herszenhorn DM. Illegal Immigrants Could Not Buy Insurance on New 'Exchange,' White House Says. Prescriptions: The Business of Health Care: New York Times; 2009.

27. Dooren J. U.S. to Spend $\$ 1$ Billion on H1N1 Flu Vaccine Production. The Wall Street Journal. 2009.

28. Wishnie MJ. Prohibiting the Employment of Unauthorized Immigrants: The Experiment Fails. U. Chi. Legal F. 2007:193.

29. Martin SF. US Immigration Reform: A System for the 21st Century. Rice University Baker Institute Latin America Initiative. 2013.

30. Rahima Jamal MG, Jean-Francois Naud, Pierre-Paul Turgeon \& Martine Leblanc. Permanent renal failure induced by pentastarch. NDT Oxford Journals. 2008.

31. Donvan J. The FDA's Caution is Hazardous to Our Health [Internet]: NPR; 2013. Podcast: 50:43

32. Zuckerman DM, Brown P, Nissen SE. Medical device recalls and the FDA approval process. Archives of internal medicine. 2011;171(11):1006.

33. Carpenter D. Policy reform: Strengthen and stabilize the FDA. Nature. 2012;485(7397):169-170. 